\title{
Size-dependent Optical Response of Magneto-plasmonic Core-shell Nanoparticles
}

\author{
Pradeep Bhatia, S.S. Verma*, and M.M. Sinha \\ Sant Longowal Institute of Engineering and Technology, Longowal-148106, Punjab (India) \\ * Corresponding author email: ssvermaus2001@yahoo.com
}

Received: 30 September 2017 / Revised: 07 October 2017 / Accepted: 09 October 2017 / Published: 09 October 2017

\begin{abstract}
ABSTRA C T
Functional properties tunability prospect of multi-layered and multi-metallic nanoparticles with respect to their emerging applications has always been the area of attraction. Magneto-plasmonic nanoparticles (MPNPs) present the possibility to exhibit their tuneable magnetic and optical properties with broad applications in magnetic resonance imaging (MRI), cancer therapy, photovoltaic solar cells, biological microscopy, optical imaging, and biosensing. Present studies found that the optical properties (absorption and scattering efficiencies) of MPNPs using $\mathrm{Fe}$, Co and Ni as magnetic materials coated with single noble metal ( $\mathrm{Au}$ or $\mathrm{Ag}$ ) or double (both $\mathrm{Au} \& \mathrm{Ag}$ ) coating layer, can be effectively tuned with the controlled size of core and shell layers. It is found that absorption and scattering LSPR peaks occur in Ultraviolet (UV) and visible region of EM for single core-shell MNP@Ag nanoparticles respectively. The absorption LSPR are found in the visible region, and scattering LSPR in NIR region and also get blue shifted for single core-shell of MNP@Au nanospheres. In case of double core-shell layers of MNP@Ag@Au nanoparticles, the absorption and scattering LSPR peaks show spectra in visible and NIR region of EM spectrum and the absorption LSPR peak shifts from visible to UV region and scattering LSPR are found in NIR region with broadening peaks of EM spectrum. It is concluded that the LSPR peak depends upon the thickness of the shell, size of core and material. The shifting of optical peaks towards the longer wavelengths depends on gold shell thickness whereas optical efficiencies depend on silver (as core or shell) thickness.
\end{abstract}

Keywords: Magneto-plasmonic nanoparticles (MPNPs), Core-shell thickness, Absorption and scattering efficiency.

\section{Introduction}

Nanoparticles (NPs) with dimensions of $1 \mathrm{~nm}$ to $100 \mathrm{~nm}$ have attracted significant interest due to their fascinating properties viz. optical, magnetic and optoelectronic that are superior to their bulk counterparts because of large surface area to volume ratio [1,2]. Nanoparticles have interesting applications in the fields of catalysis, bio-imaging, drug targeting and sensing. Significant progress has also been made in the synthesis of nanoparticles from any kinds of materials with the desired quality, quantity, and yield required for the systematic investigation of their peculiar properties and applications. Many metals (e.g., alkali metals and to some extent noble metals viz. Au and $\mathrm{Ag}$ ) can be treated as free-electron systems whose electronic and 
optical properties are determined by the conduction electrons alone. The collective oscillations of free conduction electrons (LSPR) of metallic NPs, (e.g., Ag and Au) are observed when illuminated with the light of plasmon wavelength and is called localized surface plasmon resonance (LSPR) [3]. The features of an LSPR depend upon the dispersive property of the material and its interaction with the surroundings. Metallic nanoparticles exhibiting localized surface plasmon resonances can show strong optical resonance for visible and near-infrared (NIR) wavelengths. Noble metal NPs have received considerable interest during the last few decades in the field of plasmonic, material sciences and biomedicine, due to their unique and intense optical, electronic, catalytic and tunable plasmonic properties [4-9].

On the other hand, magnetic nanoparticles (MNPs) have lesser free electron contribution to the optical properties as compared to plasmonic NPs, due to which they do not exhibit LSPR, but these MNPs possess spontaneous magnetization. Magnetic nanoparticles provide target ability or control over a motion which makes their use in a biosensor, magnetic fluids, information storage, magnetic bioseparation, and catalysis [10-16]. Recently, a great interest has arisen towards the hybrid structures of NPs to investigate their optical properties. The hybrid NPs can present enhanced novel features unavailable from single-element NPs. The advantage of hybrid NPs, compared to their single component counterparts, can be achieved in two ways. First, they can offer multiple functionalities (by coating) and secondly through coupling between their different components (alloying) by combining different classes of physical properties related to their constituents [17-19]. Corporate examples are binary (Au-Co, Ag-Co, Ag-Ni) or ternary (Fe-Ag-Si) magnetoplasmonic NPs with unique magneto-optical properties for various applications.

Magnetic nanoparticles are often used in diagnostic [20] or drug delivery purposes [21], however, they can't be used directly for in-vivo biological applications because they are sensitive to agglomeration and are corrosive. Magnetic nanoparticles can be protected by using noble metal coating, leading to core-shell nanoparticles called magneto-plasmonic nanoparticles (MPNPs). The coating not only protects the magnetic nanoparticles from oxidation, thus enhancing their stability and compatibility, but also provides a new platform for further functionalization of nanoparticles for optical properties [22-23]. The main advantage with MPNPs is to use the magnetic and plasmonic properties with an external magnetic field to provide boost to the local EM field and thus enhancing magneto-optic effects. The available experimental methods to coat magnetic nanoparticles are by directly depositing noble metals onto the core surface or by using a chelating material between the core and the shell. The optical properties of MPNPs are varied and can be tuned by changing their size, shell thickness, shapes and dielectric environment of particles [24]. Thus, the calculated optical properties (scattering and absorption efficiencies) are the relevant quantities for real applications of MPNPs.

This paper highlights the absorption and scattering efficiencies of MPNPs of single core-shell and multiple core-shell nanospheres considering $\mathrm{Fe}, \mathrm{Co}$, and $\mathrm{Ni}$ as magnetic materials at core and $\mathrm{Au}$ and $\mathrm{Ag}$ noble metal nanoparticles as coatings (shells). We found that the absorption and scattering efficiencies of considered single and multi core-shell MPNPs depend on core-shell ratio and thus can be tuned. The calculated spectra of efficiencies are found in visible and near-infrared (NIR) region of electromagnetic spectrum.

\section{Calculation Methods}

To investigate the optical properties (absorption and scattering) of nanoparticles with light (electromagnetic radiation) interaction, one has to obtain a solution to Maxwell's equations with corresponding boundary conditions on the spherical surface and the solution to the scattering problem for homogeneous sphere was proposed by Gustav Mie in 1908. Mie theory is the first analytical solution for the Maxwell equations to describe the scattering of light by spherical particles made of arbitrary materials. Mie assumed that optical constants of metals, which were obtained for bulk materials, are also applicable to nanoparticles which were at least one order of magnitude smaller than the wavelength of light, and luckily this was almost right for the size, wavelength and the material he considered. Mie theory takes less time, is cost effective and gives the same results as compared to other methods. However, for complex geometries there are other methods such as BEM, FEM, DDA and FDTD which are frequency and time domain numerical method. For the 
Bhatia et al., Adv. Nan. Res.; Vol. 1 Issue 1, pp: 1-13, 2018

spherical $\mathrm{Au}$ and $\mathrm{Ag}$ nanoparticles, $\mathrm{Q}_{\mathrm{abs}}$ and $\mathrm{Q}_{\mathrm{sca}}$ were calculated on the basis of Mie theory for homogeneous spheres. The Mie total extinction $\left(Q_{\text {ext }}\right)$ and scattering efficiency $\left(Q_{\text {sca }}\right)$ for a homogeneous sphere are expressed as infinite series:

$$
\begin{gathered}
Q_{e x t}=\frac{2}{x^{2}} \sum_{n=1}^{\infty}(2 n+1) \operatorname{Re}\left[a_{n}+b_{n}\right] \\
Q_{s c a}=\frac{2}{x^{2}} \sum_{n=1}^{\infty}(2 n+1)\left[a_{n}^{2}+b_{n}^{2}\right] \\
a_{n}=\frac{m \varphi_{n}(m x) \varphi_{n}^{\prime}(x)-\varphi_{n}(x) y_{n}^{\prime}(m x)}{m \varphi_{n}(m x) \varepsilon_{n}^{\prime}(x)-m \varepsilon_{n}(x) \varphi_{n}^{\prime}(m x)} \\
b_{n}=\frac{\varphi_{n}(m x) \varphi_{n}^{\prime}(x)-m \varphi_{n}(x) \varphi_{n}^{\prime}(m x)}{\varphi_{n}(m x) \varepsilon_{n}^{\prime}(x)-m \varepsilon_{n}(x) \varphi_{n}^{\prime}(m x)}
\end{gathered}
$$

where $m$ is the ratio of refractive index of the sphere $n$ to that of the surrounding medium $n_{m}, x$ is the size parameter given as $2 \pi n_{m} R / \lambda, \varphi_{n}$ and $\varepsilon_{n}$ are the Riccati-Bessel functions, and the prime represents first differentiation with respect to the argument in parentheses. Numerical calculations of the Mie series are performed at discrete points in the wavelength range from 200 to $1200 \mathrm{~nm}$.

Calculations of the optical properties such as absorption and scattering efficiency of considered core-shell nanoparticles were performed by using a computer code employing Mie scattering for multi-layered spherical geometry developed by Ovidio Pena-Rodriguez team [25]. Mielab offers tools to perform several tasks related to the calculation of optical properties of nanoparticles and particles ensembles. Input parameters are the thickness of each layer, real (n) and imaginary $(\mathrm{k})$ parts of the materials complex refractive index corresponding to energy in electron volt $(\mathrm{eV})$, and the last parameter is the refractive index of the surrounding medium. The complex refractive index of water is $1.33+0 \mathrm{i}$ chosen as a surrounding medium to MPNPs because it is the main component of environments encountered in biomedical applications of MPNPs. The complex dielectric constant of magnetic and plasmonic (noble) material is obtained from Johnson and Christy [26-27]. The thickness or size of core-shell layers are not represented by the single radius, but are defined by the total radius $\left(\mathrm{R}_{\text {total }}\right)$ for the structure, and given as the sum of the radii of the magnetic core $\left(\mathrm{R}_{\text {core }}\right)$ and noble metal shell ( $\left.\mathrm{R}_{\text {shell }}\right)$. Plasmonics properties of core-shell nanoparticles are calculated for two sets of the nanostructure, firstly: by fixing the core and varying shell thickness for the total size and then changing the size of the core and varying the shell size also in total size. Secondly, by changing the core size and shell size as in the first case and fixing the size of the outer shell for total size.

\section{Results and Discussion}

Presently, optical properties are represented by scattering $\left(\mathrm{Q}_{\mathrm{sca}}\right)$ and absorption $\left(\mathrm{Q}_{\mathrm{abs}}\right)$ efficiencies. Since different optical properties of MPNPs are only associated with scattering and absorption efficiencies, therefore, these are the relevant representative parameters for real applications of MPNPs. Therefore, we have calculated the optical spectra for these important parameters $\left(\mathrm{Q}_{\text {sca }}\right.$ and $\left.\mathrm{Q}_{\mathrm{abs}}\right)$ of core-shell magneticplasmonic nanoparticles with the different core (Fe, Co, Ni)-shell (Ag, $\mathrm{Au}$ ) materials and thickness, considering nanospheres of various sizes between $20 \mathrm{~nm}$ to $70 \mathrm{~nm}$. 


\subsection{Single Core-shell MPNPs}

In the single core-shell study of nanospheres, we studied the magnetic nanospheres of $\mathrm{Fe}$, $\mathrm{Co}$ and $\mathrm{Ni}$ coated with $\mathrm{Ag}$ or $\mathrm{Au}$ or both plasmonic materials. The optical properties were calculated as a function of wavelength, starting with the smallest size of $10 \mathrm{~nm}$ of the core and then changing the shell thickness from $10 \mathrm{~nm}$ to $60 \mathrm{~nm}$. The core size was changed from $10 \mathrm{~nm}$ to $60 \mathrm{~nm}$ and the thickness of the shell was varied to complete the total size of core-shell up to $70 \mathrm{~nm}$ of considered nanospheres. We considered the maximum-height and -broadening of peak to optimize the shell thickness. In this way, we optimize the shell thickness for each considered core size of nanospheres.

\subsubsection{MNP@Ag Nanoparticles}

Figure 1(a) represents the LSPR peaks for Fe@Ag core shells and only the maximum peaks for varying core size and shell thickness have been shown. The core size is considered to be increasing from $10 \mathrm{~nm}$ to $60 \mathrm{~nm}$ with an increment of $10 \mathrm{~nm}$. The LSPR peak is found at $413 \mathrm{~nm}$ for $10 \mathrm{~nm}$ core with $20 \mathrm{~nm}$ shell thickness and other peaks are at $382 \mathrm{~nm}, 362 \mathrm{~nm}, 361 \mathrm{~nm}, 365 \mathrm{~nm}$ and $360 \mathrm{~nm}$ for 20-60 nm core size with $10 \mathrm{~nm}$ shell thickness for each core size. As the size of shell increases for each considered core size, the peak position shifts towards the higher wavelength with a decrease in efficiency. With the increase in core size the peak position shifts to lower wavelength from $382 \mathrm{~nm}$ to $360 \mathrm{~nm}$ as shown in Figure 1(a). Thus, the LSPR absorption peaks show the spectra near Ultraviolet (UV) regime in electromagnetic (EM) spectrum.

Figure 1(b) shows absorption spectra representing various peaks of Co@Ag at wavelength $414 \mathrm{~nm}$ for 10 $\mathrm{nm}$ core sizes with $20 \mathrm{~nm}$ shell thickness, and $396 \mathrm{~nm}, 366 \mathrm{~nm}, 367 \mathrm{~nm}, 374 \mathrm{~nm}, 370 \mathrm{~nm}$ for $10 \mathrm{~nm}$ shell thickness of remaining 20 to $60 \mathrm{~nm}$ core size respectively. As the size of shell increases for each considered core size, the peak position shifts toward the higher wavelength with a decrease in efficiency. With an increase in core size, the peak position shifts to a lower wavelength from $414 \mathrm{~nm}$ to $366 \mathrm{~nm}$. Thus, the LSPR absorption peaks show the spectra near the UV regime in EM spectrum. Similarly, for Ni@Ag coreshell, we get various peaks at wavelength $415 \mathrm{~nm}$ for $20 \mathrm{~nm}$ shell thickness, and $405 \mathrm{~nm}, 438 \mathrm{~nm}, 369 \mathrm{~nm}$, $377 \mathrm{~nm}, 393 \mathrm{~nm}$ for $10 \mathrm{~nm}$ shell thickness of each considered 10 to $60 \mathrm{~nm}$ core size respectively. As the shell thickness increases for each considered core size, the peak position shifts toward the higher wavelength with decreasing efficiency. With the increase in core size, the peak position shifts to a lower wavelength between $415 \mathrm{~nm}$ and $369 \mathrm{~nm}$ as shown in Figure 1(c). Thus, the overall LSPR absorption peaks for MPNPs show the spectra near the UV regime on EM spectrum.

The scattering efficiencies of MNPs@Ag core-shell have also been calculated, and we get various peaks at wavelengths $580 \mathrm{~nm}, 580 \mathrm{~nm}, 580 \mathrm{~nm}, 583 \mathrm{~nm}, 583 \mathrm{~nm}, 580 \mathrm{~nm}$ for 10 to $60 \mathrm{~nm}$ core-shells respectively for Fe@Ag magneto-plasmonic nanoparticles as shown in Figure 1(d). With the increase in shell thickness for considered core size, the peak position shifts toward the higher wavelength with a decrease in efficiency. Thus, the LSPR scattering peak shows the spectra in the visible region of electromagnetic spectrum. Similarly, Figure 1(e) shows various peaks of Co@Ag at wavelengths 580 nm, 580 nm, 580 nm, 583 nm, $583 \mathrm{~nm}$, and $580 \mathrm{~nm}$ for 10 to $60 \mathrm{~nm}$ core sizes with shell thickness $60 \mathrm{~nm}$ to $10 \mathrm{~nm}$ respectively. As the shell thickness increases for considered core size, the peak position shifts toward the higher wavelength with a decrease in efficiency. With the increase in core size, the peak position shifts to higher wavelength from $580 \mathrm{~nm}$ to $583 \mathrm{~nm}$. Thus, the LSPR scattering spectra is found in the visible region of electromagnetic spectrum. Further, for $\mathrm{Ni@Ag} \mathrm{core-shell,} \mathrm{we} \mathrm{get} \mathrm{various} \mathrm{peaks} \mathrm{at} \mathrm{wavelengths} 580 \mathrm{~nm}, 580 \mathrm{~nm}, 583 \mathrm{~nm}$, $583 \mathrm{~nm}, 586 \mathrm{~nm}$, and $590 \mathrm{~nm}$ for $10 \mathrm{~nm}$ to $60 \mathrm{~nm}$ core sizes with shell thickness $60 \mathrm{~nm}$ to $10 \mathrm{~nm}$ respectively. As the shell thickness increases for each considered core size, the peak position shifts toward the higher wavelength with a decrease in efficiency. With the increase in core size and decrease in shell thickness the peak position shifts to higher wavelength from $580 \mathrm{~nm}$ to $590 \mathrm{~nm}$ as shown in Figure 1(f). Thus, the LSPR scattering peak shows the spectra in the visible region of EM spectrum. Furthermore, the Figure $1(\mathrm{~g}, \mathrm{~h})$ give relation between core size and LSPR wavelength of single and multi core-shell 
Bhatia et al., Adv. Nan. Res.; Vol. 1 Issue 1, pp: 1-13, 2018

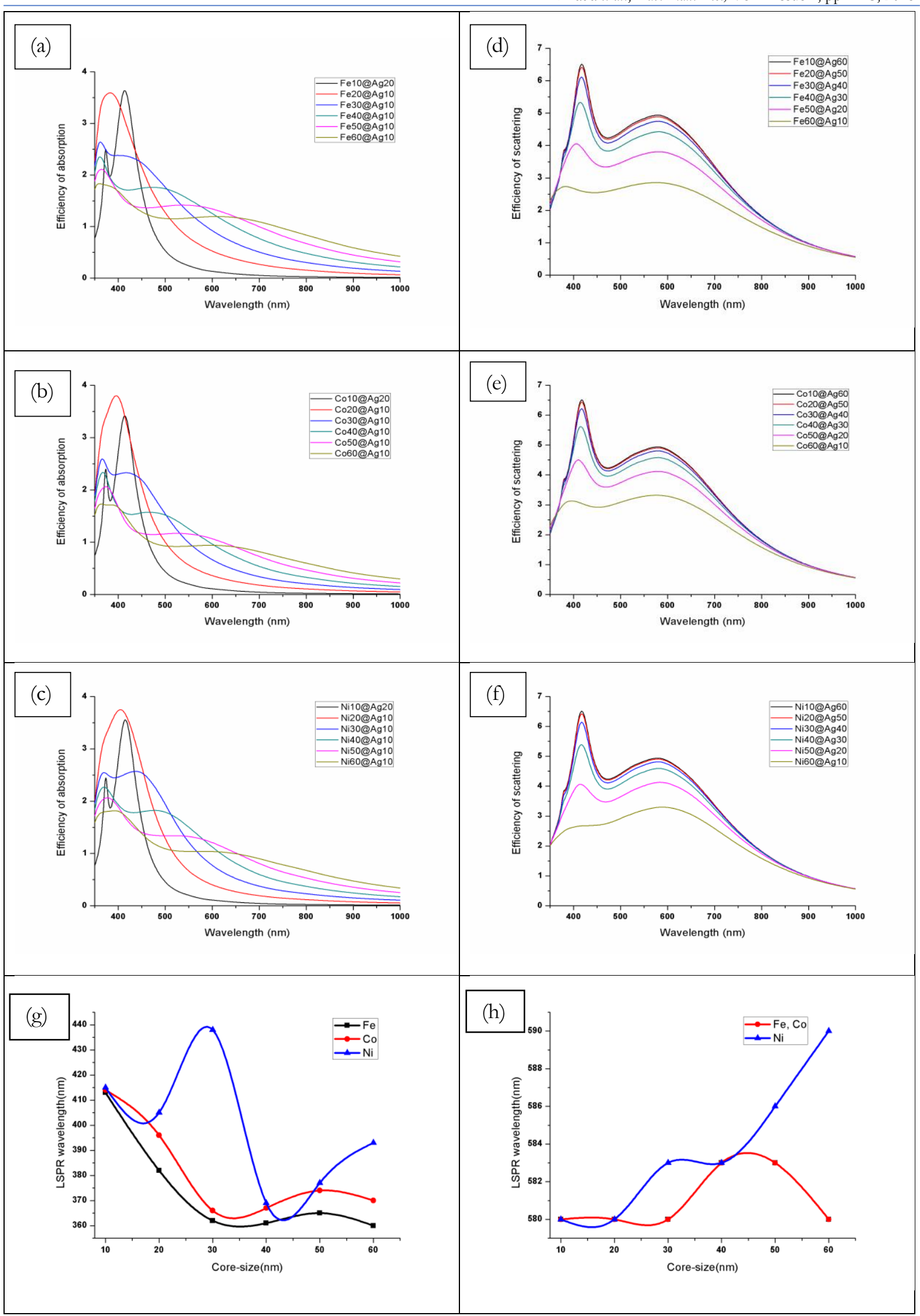

Figure 1: Calculated absorption efficiencies (a) Fe@Ag (b)Co@Ag (c) Ni@Ag and scattering efficiencies (d) $F e @ A g(e) C o @ A g(f) N i @ A g$ and size dependent LSPR wavelength $(g, h)$ for single core-shell nanospheres of magneto-plasmonics. 
Size-dependent Optical Response of Magneto-plasmonic Core-shell Nanoparticles

nanostructures for absorption (Figure 1g) and scattering (Figure 1h) to represent distinct LSPR tunability with considered core-shell thickness. Further analysed, there is an occurrence of two LSPR peaks in Figure1; the first peak appears at lower wavelength and the second at higher wavelength which can be accounted towards the dipole and quadrupole resonant mode contribution in the LSPR spectra. Since, the interaction between incident energy with particles produces the external driving field which is not uniform. Therefore, dynamic polarization happens in the particle and second order LSPR such as quadrupole resonant mode will contribute to LSPR spectra.

\subsection{2ＭNP@Au Nanoparticles}

The spectra of absorption and scattering efficiency have been calculated for gold ( $\mathrm{Au}$ ) coated on $\mathrm{Fe}$, $\mathrm{Co}$ and Ni spherical nanostructures. The absorption peaks for Fe@Au core-shell nanospheres have been calculated from 10 to $60 \mathrm{~nm}$ size of core at wavelengths $541 \mathrm{~nm}$ of $30 \mathrm{~nm}$ shell thickness, $534 \mathrm{~nm}$ of 20 $\mathrm{nm}$ shell thickness, $532 \mathrm{~nm}$ of $20 \mathrm{~nm}$ shell thickness, and $521 \mathrm{~nm}, 517 \mathrm{~nm}, 507 \mathrm{~nm}$ of $10 \mathrm{~nm}$ shell thickness respectively shown in Figure 2(a). As the size of core increases with the combination of shell thickness, the peak position shifts to lower wavelength from $541 \mathrm{~nm}$ to $507 \mathrm{~nm}$. Thus, LSPR absorption peaks are found in the visible region of EM spectrum. Figure 2(b) shows various peaks of Co@Au at wavelengths $541 \mathrm{~nm}$ for 10 and $30 \mathrm{~nm}, 534 \mathrm{~nm}$ for 20 and $20 \mathrm{~nm}, 532 \mathrm{~nm}$ for 30 and $20 \mathrm{~nm}$, and $519 \mathrm{~nm}, 510 \mathrm{~nm}, 501 \mathrm{~nm}$ for $40 \mathrm{~nm}$ to $60 \mathrm{~nm}$ size of core with shell thickness $10 \mathrm{~nm}$ respectively. As the size of core increases with the combination of shell thickness, the peak position shifts from $541 \mathrm{~nm}$ to $507 \mathrm{~nm}$. Thus, LSPR absorption peaks are found in the visible region of EM spectrum. For Ni@Au core-shell, we get various peaks at wavelengths $542 \mathrm{~nm}$ for 10 and $30 \mathrm{~nm}, 536 \mathrm{~nm}$ for 20 and $20 \mathrm{~nm}, 538 \mathrm{~nm}$ for 30 and $20 \mathrm{~nm}$, and $525 \mathrm{~nm}$, $516 \mathrm{~nm}, 505 \mathrm{~nm}$ for $40 \mathrm{~nm}$ to $60 \mathrm{~nm}$ size of the core with shell thickness $10 \mathrm{~nm}$ respectively. As the size of core increases with the combination of shell thickness, the peak position shifts from $542 \mathrm{~nm}$ to $505 \mathrm{~nm}$ as shown in Figure 2(c). Thus, LSPR absorption peaks are found in the visible region of EM spectrum.

We get the scattering peaks for Fe@Au at wavelengths 636 nm, 636 nm, 636 nm, 634 nm, 630 nm, and 613 $\mathrm{nm}$ for $10 \mathrm{~nm}$ to $60 \mathrm{~nm}$ core sizes with shell thickness $60 \mathrm{~nm}$ to $10 \mathrm{~nm}$ respectively shown in Figure 2(d). As the thickness of shell increases for each considered core size, the peaks position shifts to the higher wavelength with greater efficiency. The scattering peaks are found in NIR of EM spectrum, and peaks are shifted from $636 \mathrm{~nm}$ to $613 \mathrm{~nm}$ with increase in core size. Thus, LSPR peaks get blue shifted for Fe@Au core-shell nanospheres. For Co@Au core-shell, the various peaks are found at wavelengths $636 \mathrm{~nm}, 636$ $\mathrm{nm}, 636 \mathrm{~nm}, 633 \mathrm{~nm}, 629 \mathrm{~nm}, 614 \mathrm{~nm}$ for $10 \mathrm{~nm}$ to $60 \mathrm{~nm}$ core sizes with shell thickness $60 \mathrm{~nm}$ to $10 \mathrm{~nm}$ respectively. As the thickness of shell increases for each considered core size, the peak position shifts to higher wavelength with greater efficiency as shown in Figure 2(e). With the increase in core size, peaks shift from $636 \mathrm{~nm}$ to $614 \mathrm{~nm}$ and the spectra are found in NIR on EM spectrum. Thus, LSPR peaks get blue shifted for Co@Au core-shell nanospheres. Figure 2(f) shows various peaks of Ni@Au at wavelengths 636 $\mathrm{nm}, 636 \mathrm{~nm}, 636 \mathrm{~nm}, 636 \mathrm{~nm}, 623 \mathrm{~nm}$, and $625 \mathrm{~nm}$ for $10 \mathrm{~nm}$ to $60 \mathrm{~nm}$ core sizes with decrease in shell thickness from $60 \mathrm{~nm}$ to $10 \mathrm{~nm}$ respectively. As the thickness of shell increases for each considered core size, the peak position shifts to higher wavelength with greater efficiency. The peak is shifting from $636 \mathrm{~nm}$ to $625 \mathrm{~nm}$ with an increase in core size and spectra are found in NIR region on EM spectrum. Thus, LSPR peaks get blue shifted for $\mathrm{Ni@Au} \mathrm{core-shell} \mathrm{nanospheres.} \mathrm{In} \mathrm{addition,} \mathrm{the} \mathrm{Figure} \mathrm{2(g,} \mathrm{h)} \mathrm{give} \mathrm{relation}$ between core size and LSPR wavelength of single and multi core-shell nanostructures for absorption (Figure $2 \mathrm{~g}$ ) and scattering (Figure $2 \mathrm{~h}$ ) to represent distinct LSPR tunability with considered core-shell thickness. 
Bhatia et al., Adv. Nan. Res.; Vol. 1 Issue 1, pp: 1-13, 2018

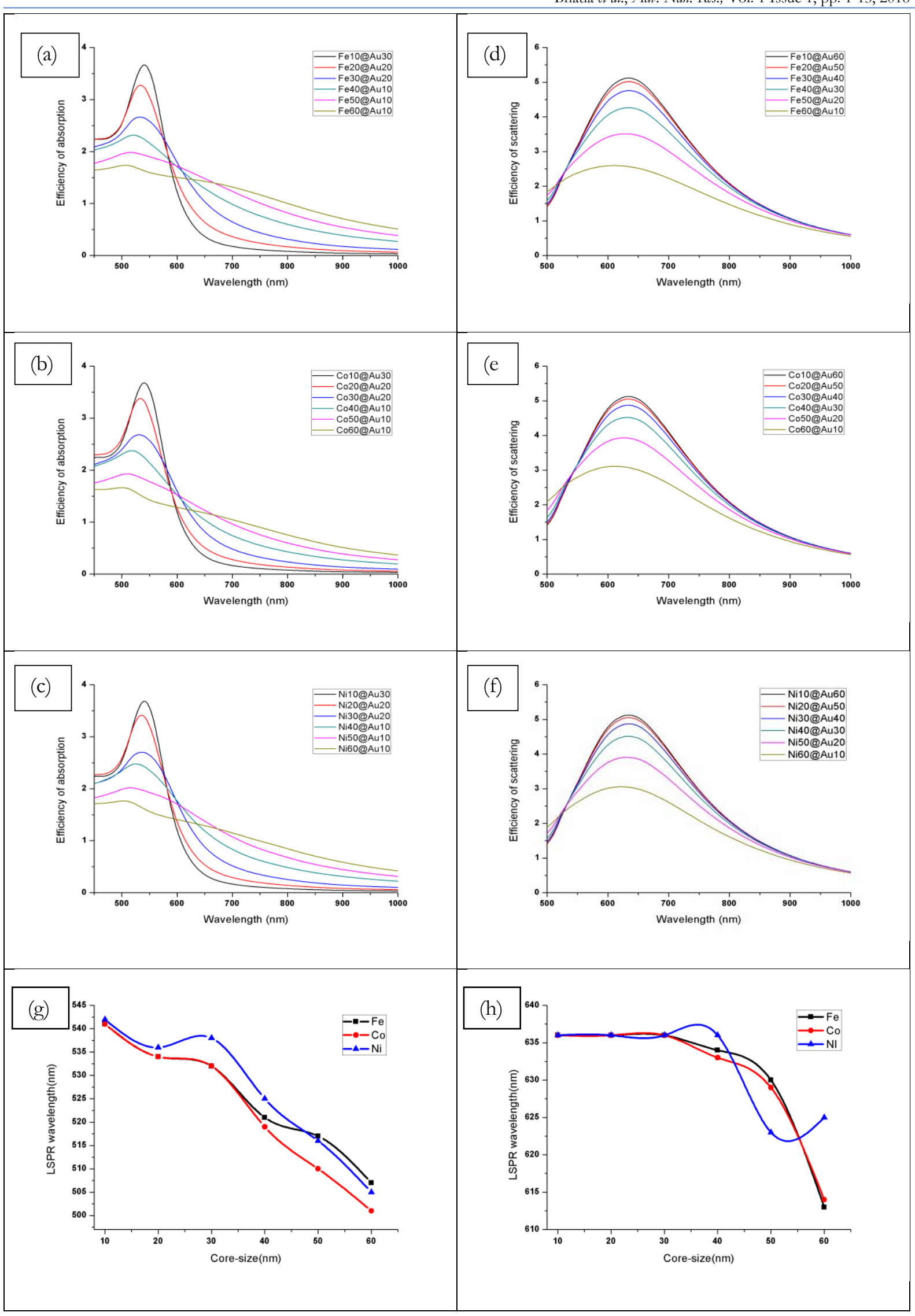

Figure 2: Calculated absorption efficiencies (a)Fe@Au (b)Co@Au (c)Ni@Au and scattering efficiencies (d) $\mathrm{Fe} @ A u(e) C o @ A u(f) N i @ A u$ and size dependent LSPR wavelength $(g, h)$ for single core-shell nanospheres of magneto-plasmonics 


\subsection{Multi-layer Core-shell MPNPs}

We coated magnetic nanoparticles of $\mathrm{Fe}$, $\mathrm{Co}$ and $\mathrm{Ni}$ firstly with $\mathrm{Ag}$ and then with $\mathrm{Au}$ nanoparticles and vice versa. We used the optimized core-shell size of single core-shell nanospheres and fixed the outer shell with $10 \mathrm{~nm}$ thickness for multi core-shell. Thus, we calculated the absorption (left side) and scattering (right side) efficiencies of $80 \mathrm{~nm}$ total size of multiple core-shell nanospheres as shown in Figures 3 and 4.

\subsection{1ＭNP@Ag@Au Nanoparticles}

Figure 3(a) shows the absorption spectra for Fe@Ag@Au multi core-shell nanospheres at wavelengths 541 $\mathrm{nm}, 541 \mathrm{~nm}, 521 \mathrm{~nm}, 504 \mathrm{~nm}, 401 \mathrm{~nm}$ and $444 \mathrm{~nm}$ for $10 \mathrm{~nm}$ to $60 \mathrm{~nm}$ core sizes with the combination of shell thickness respectively. For a $10 \mathrm{~nm}$ core size, the peak position shifts toward higher wavelength with greater absorption efficiency for 10 and $20 \mathrm{~nm}$ shell thickness, and towards lower wavelength with lesser absorption efficiency for 30 to $60 \mathrm{~nm}$ shell thickness respectively. On increasing the size of core above 20 $\mathrm{nm}$ with a combination of shell thickness, spectra are found with lesser absorption efficiency at lower wavelength. Absorption LSPR peaks are found between $541 \mathrm{~nm}$ and $401 \mathrm{~nm}$ on EM spectrum. For Co@Ag@Au multi core-shell MPNPs, various peaks are found at wavelengths $518 \mathrm{~nm}, 518 \mathrm{~nm}, 515 \mathrm{~nm}$, $502 \mathrm{~nm}, 415 \mathrm{~nm}, 442 \mathrm{~nm}$ for $10 \mathrm{~nm}$ to $60 \mathrm{~nm}$ core size respectively as shown in Figure 3(b), with the combination of shell thickness. As the core size increases, the LSPR peaks shift from $518 \mathrm{~nm}$ to $415 \mathrm{~nm}$. Various absorption efficiency spectra of $\mathrm{Ni@Ag@Au} \mathrm{nanospheres} \mathrm{at} \mathrm{wavelengths} 518$ nm, 521 nm, 519 $\mathrm{nm}, 502 \mathrm{~nm}, 422 \mathrm{~nm}, 457 \mathrm{~nm}$ for $10 \mathrm{~nm}$ to $60 \mathrm{~nm}$ core sizes with the combination of shell thickness respectively are shown in Figure 3(c). The LSPR peaks are found between $521 \mathrm{~nm}$ and $422 \mathrm{~nm}$ when the size of shell increases. Moreover, there is shifting of absorption peak towards the higher wavelength with peak difference of 100 - $150 \mathrm{~nm}$ due to the Au coating on MNP@Ag core-shell nanospheres.

The scattering efficiency spectra for Fe@Ag@Au multi core-shell nanospheres at wavelengths 657 nm, 657 $\mathrm{nm}, 657 \mathrm{~nm}, 661 \mathrm{~nm}, 663 \mathrm{~nm}$ and $665 \mathrm{~nm}$ for $10 \mathrm{~nm}$ to $60 \mathrm{~nm}$ core sizes with shell thickness $60 \mathrm{~nm}$ to 10 $\mathrm{nm}$ respectively are shown in Figure 3(d). LSPR peaks shift towards higher wavelength when the core size is less than $30 \mathrm{~nm}(10-30 \mathrm{~nm})$, and for core size greater than $40 \mathrm{~nm}$, the LSPR peaks also move towards higher wavelength with an increase in scattering efficiency. As the core size increases with decrease in the shell thickness, LSPR peaks shift between $665 \mathrm{~nm}$ and $657 \mathrm{~nm}$ and spectra are found in NIR region of EM spectrum. Figure 3(e) shows the LSPR spectra for Co@Ag@Au nanospheres at wavelengths 657 nm, 657 $\mathrm{nm}, 657 \mathrm{~nm}, 657 \mathrm{~nm}, 662 \mathrm{~nm}$ and $665 \mathrm{~nm}$ for the considered multi core-shells. For core size between 10$40 \mathrm{~nm}$, LSPR peaks shift towards higher wavelength, and also shift towards higher wavelength for $50 \mathrm{~nm}$ and $60 \mathrm{~nm}$ with an increase in efficiency. LSPR peaks shift between $665 \mathrm{~nm}$ and $657 \mathrm{~nm}$ and spectra are found in NIR region of EM spectrum when core size increases with decrease in shell thickness. In case of Ni@Ag@Au, the scattering peaks are found at wavelengths 657 nm, 657 nm, 657 nm, 659 nm, 661 nm and $664 \mathrm{~nm}$ for $10 \mathrm{~nm}$ to $60 \mathrm{~nm}$ core sizes with shell thickness $60 \mathrm{~nm}$ to $10 \mathrm{~nm}$ respectively as shown in Figure $3(\mathrm{f})$. For core size less than $40 \mathrm{~nm}(10-40 \mathrm{~nm})$, LSPR peaks shift toward higher wavelength and with core size greater than $50 \mathrm{~nm}$, LSPR peaks shift towards higher wavelength with an increase in efficiency. As the core size increases with decrease in the shell thickness, the LSPR peaks shift between $664 \mathrm{~nm}$ and $657 \mathrm{~nm}$, and spectra are found in NIR region of EM spectrum. Moreover, there is shifting of scattering peaks towards the higher wavelength with peak difference of 75 - $80 \mathrm{~nm}$ due to the Au coating on MNP@Ag core-shell nanospheres. Further, the Figure $3(\mathrm{~g}, \mathrm{~h})$ give relation between core size and LSPR wavelength of single and multi core-shell nanostructures for absorption (Figure 3g) and scattering (Figure 3h) to represent distinct LSPR tunability with considered core-shell thickness. 


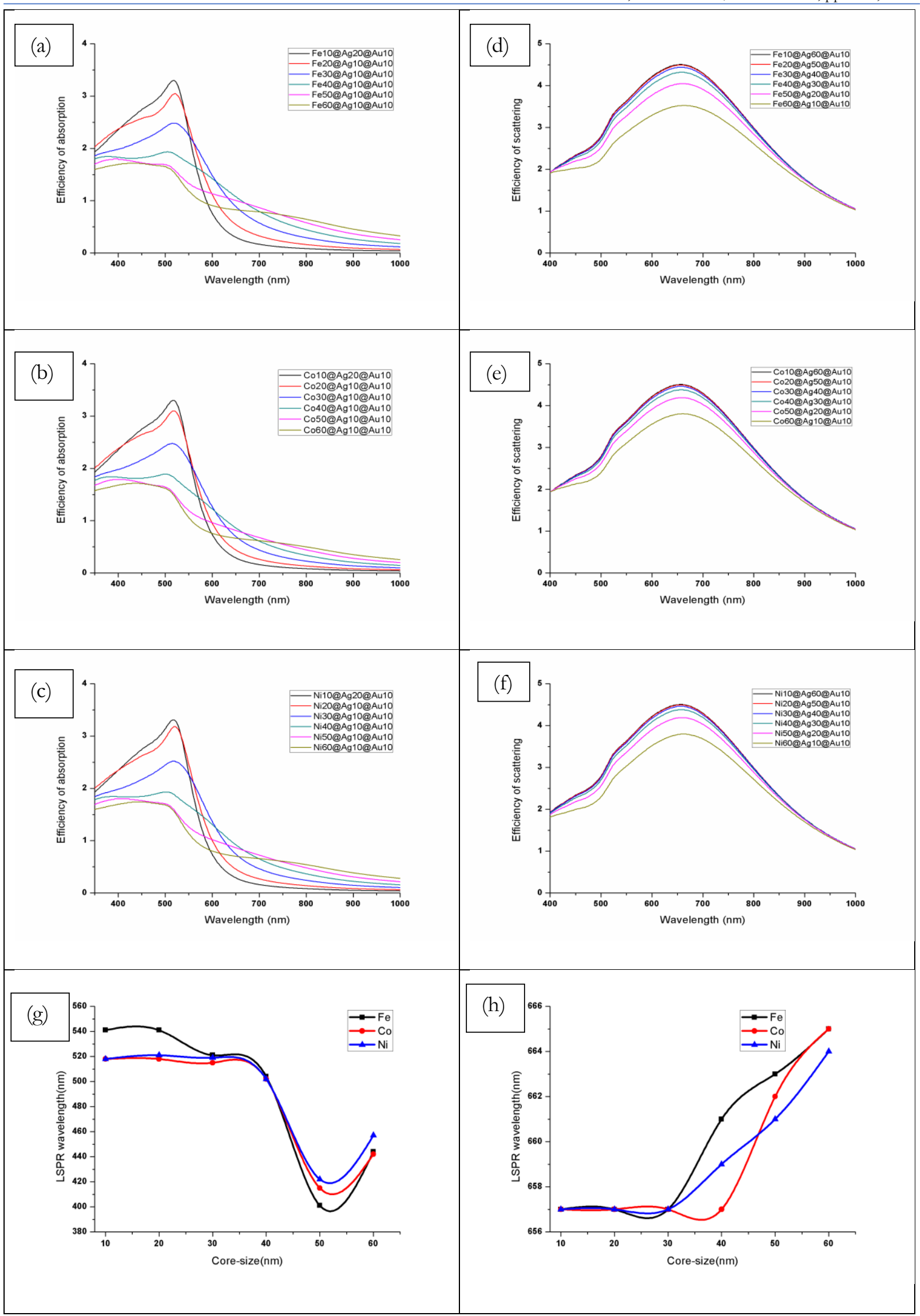

Figure 3: Calculated absorption efficiencies (a)Fe@Ag@Au (b)Co@Ag@Au (c) Ni@Ag@Au and scattering efficiencies(d)Fe@Ag@Au (e)Co@Ag@Au (f)Ni@Ag@Au and sizedependentLSPRwavelength $(g, h)$ for multiple core-shell nanospheres of magneto-plasmonics 


\subsubsection{MNP@Au@Ag Nanoparticles}

Absorption and scattering spectra have also been calculated for MNP@Au@Ag core-shell nanospheres. The absorption peaks for Fe@Au@Ag at wavelengths 487 nm, 471 nm, 496 nm, 376 nm, 439 nm and 656 $\mathrm{nm}$ for $10 \mathrm{~nm}$ to $60 \mathrm{~nm}$ core size with the combination of shell thickness respectively are as shown in Figure 4(a). On increasing the core size for different combinations of shell thickness, the spectra are found between $656 \mathrm{~nm}$ and $376 \mathrm{~nm}$, in the NIR to UV region of EM spectrum. For Co@Au@Ag, the absorption peaks are found at wavelengths $490 \mathrm{~nm}, 462 \mathrm{~nm}, 493 \mathrm{~nm}, 382 \mathrm{~nm}, 442 \mathrm{~nm}$ and $430 \mathrm{~nm}$ for $10 \mathrm{~nm}$ to 60 $\mathrm{nm}$ core size with the combination of considered shell thickness respectively. As the core size increases for various combinations of shell thickness, the spectra are found between $493 \mathrm{~nm}$ and $382 \mathrm{~nm}$ wavelength with broadening peaks as shown in Figure 4(b). Hence, the absorption LSPR is found from visible to UV region of EM spectrum. Figure 4(c) shows the peaks for Ni@Au@Ag nanospheres at wavelengths 487 nm, $469 \mathrm{~nm}, 496 \mathrm{~nm}, 382 \mathrm{~nm}, 442 \mathrm{~nm}$ and $430 \mathrm{~nm}$ for the considered multi core-shell. The LSPR spectra are found between $496 \mathrm{~nm}$ and $382 \mathrm{~nm}$ with broadening of the peak when the core size increases along with the various values of shell thickness. Thus, the LSPR absorption peaks shift from visible to UV region of EM spectrum. Lastly, it has to be mentioned that due to Ag coating on MNP@Au core-shell nanospheres, broadening in scattering peaks have been found.

Scattering peaks for Fe@Au@Ag multiple core-shell are found at wavelengths 656 nm, 656 nm, 656 nm, $657 \mathrm{~nm}, 658 \mathrm{~nm}$ and $656 \mathrm{~nm}$ for $10 \mathrm{~nm}$ to $60 \mathrm{~nm}$ core size with shell thickness $60 \mathrm{~nm}$ to $10 \mathrm{~nm}$ respectively as shown in Figure 4(d). For core size less than $30 \mathrm{~nm}(10-30 \mathrm{~nm})$, LSPR peaks shift towards higher wavelength, and for core size more than $40 \mathrm{~nm}$, LSPR peaks shift towards higher wavelength with an increase in efficiency. As the core size increases with decrease in shell thickness, LSPR peaks are found in NIR region of EM spectrum. Figure 4(e) shows the various peaks for Co@Au@Ag nanospheres at wavelengths $654 \mathrm{~nm}, 654 \mathrm{~nm}, 654 \mathrm{~nm}, 655 \mathrm{~nm}, 656 \mathrm{~nm}$ and $654 \mathrm{~nm}$ for $10 \mathrm{~nm}$ to $60 \mathrm{~nm}$ core sizes with Au shell thickness $60 \mathrm{~nm}$ to $10 \mathrm{~nm}$ respectively and fixed outer Ag shell thickness of $10 \mathrm{~nm}$. For core size less than $30 \mathrm{~nm}$ (10-30 nm), LSPR peaks shift towards higher wavelength, and for core size greater than $40 \mathrm{~nm}$, LSPR peaks shift towards higher wavelength and efficiency increases. With the increasing core size and decreasing shell thickness, LSPR peaks are found in NIR region of EM spectrum. For Ni@Au@Ag MPNPs, the scattering peaks are shown in Figure 4(f) at wavelengths $655 \mathrm{~nm}, 655 \mathrm{~nm}, 655 \mathrm{~nm}, 655 \mathrm{~nm}$, $657 \mathrm{~nm}$, and $654 \mathrm{~nm}$ for the considered multi core-shell thickness. For the core size less than 30nm (10$30 \mathrm{~nm}$ ), LSPR peaks shift towards higher wavelength, and for core size greater than 40nm, LSPR peaks shift towards higher wavelength with an increase in efficiency. On increasing core size with decrease in shell thickness, LSPR peaks shift between $654 \mathrm{~nm}$ and $657 \mathrm{~nm}$ and LSPR spectra is found in NIR region of EM spectrum. Moreover, with Ag coating on MNP@Au core-shell nanospheres broadening of peaks has been observed along with shifting of peaks towards higher wavelength with peak difference of $15-20 \mathrm{~nm}$.

Similarly, the Figure 4(g, h) give relation between core size and LSPR wavelength of single and multi coreshell nanostructures for absorption (Figure 4g) and scattering (Figure 4h) to represent distinct LSPR tunability with considered core-shell thickness. 


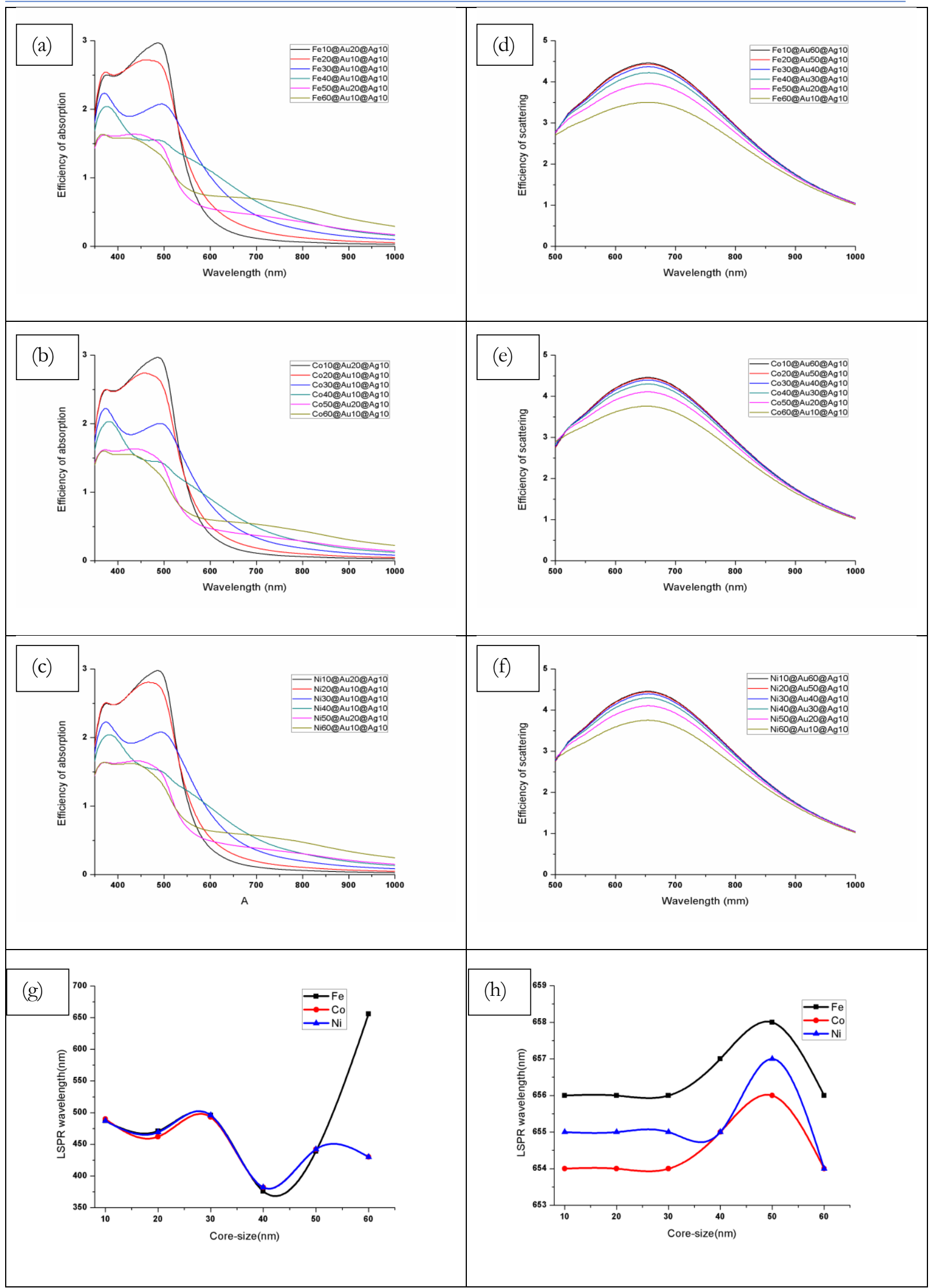

Figure 4: Calculated absorption efficiencies (a)Fe@Au@Ag (b)Co@Au@Ag (c)Ni@Au@Agand scattering efficiencies (d)Fe@Au@Ag (e)Co@Au@Ag (f)Ni@Au@Ag and sizedependent LSPR wavelength $(g, h)$ for multiple core-shell nanospheres of magneto-plasmonics. 


\section{Conclusion}

We calculated the optical properties (absorption and scattering) of single and multi core-shell magnetoplasmonic nanoparticles (MPNPs) with Fe, Co and Ni as core materials and $\mathrm{Ag}$ or/and $\mathrm{Au}$ as shell materials with water as a surrounding medium to control the LSPR peaks. It was found that absorption and scattering LSPR peaks are in UV and visible region of EM for core-shell MNP@Ag nanospheres respectively. The absorption LSPR peaks are found in visible region and scattering LSPR peaks in NIR region along with blue shift for MNP@Au nanospheres and these peaks show spectra in visible and NIR region of EM spectrum with a shifting of absorption peaks from visible to UV region and scattering LSPR are found in NIR region with broadening peaks on EM spectrum for multi core-shells of MNP@Au@Ag nanospheres. It is found that shifting of optical peaks towards higher wavelengths depends on gold shell thickness whereas optical efficiencies depend on silver (as core or shell) thickness. It is also observed that doublelayered MPNPs show larger LSPR peaks for scattering and absorption as compared to single core-shell MPNPs. Thus, it is concluded that the LSPR peak depends upon the thickness of the shell, size of core and material. Tuning and controlling the optical properties of MPNPs in the UV to NIR region of the electromagnetic spectrum may be useful for the applications of MNPs in optical imaging and cancer therapy.

\section{Acknowledgments}

The author, Pradeep Bhatia, would like to thank Ovidio Pena-Rodriguez, Pedro Pablo Gonzalez-Perez and Umapada Pal towards the use of Mielab software (freely downloaded from internet). I am also very thankful to S.L.I.E.T. Longowal for providing financial assistance and computational facilities towards my Ph.D.

\section{How to Cite this Article:}

P. Bhatia, S. S. Verma, and M. Sinha, "Size-dependent Optical Response of Magneto-plasmonic Core-shell Nanoparticles”, Adv. Nan. Res., vol. 1, no. 1, pp. 1-13, Oct. 2017. doi: 10.21467/anr.1.1.1-13

\section{References}

[1] M.Ventra, S. Evoy and J.R. Heflin (Eds.), Introduction to nanoscale science and technology, Springer Science \& Business Media, 2006.

[2] X. Huang, S. Neretina and M.A.El-Sayed, "Gold nanorods: from synthesis and properties to biological and biomedical applications", Advanced Materials, 21(48), 4880-4910, 2009.

[3] C.F. Bohren and D.R.Huffman, Absorption and scattering of light by small particles, John Wiley \& Sons, 2008.

[4] S.A. Maier, Plasmonics: fundamentals and applications, Springer Science \& Business Media, 2007.

[5] S.M.Silva, R. Tavallaie, L.Sandiford, R.D. Tilley and J.J. Gooding, "Gold coated magnetic nanoparticles: from preparation to surface modification for analytical and biomedical applications", Chemical Communications, 52(48), 7528-7540, 2016.

[6] J. Lim and S.A. Majetich, S. A., "Composite magnetic-plasmonic nanoparticles for biomedicine: manipulation and imaging", Nano Today, 8(1), 98-113, 2013.

[7] J.N.Anker, W.P. Hall, O. Lyandres, N.C.Shah, J. Zhao and R.P. Van Duyne, R. P., "Biosensing with plasmonic nanosensors", Nature materials, 7(6), 442-453, 2008.

[8] A. Wu, P. Ou and L. Zeng, L., "Biomedical applications of magnetic nanoparticles", Nano, 5(05), 245-270, 2010.

[9] T.R. Jensen, M.D. Malinsky, C.L.Haynes and R.P.Van Duyne, R. P., "Nanosphere lithography: tunable localized surface plasmon resonance spectra of silver nanoparticles", The Journal of Physical Chemistry B, 104(45), 10549-10556, 2000.

[10] M.M.Miller, G.A. Prinz, S.F.Chen and S. Bounnak, "Detection of a micron-sized magnetic sphere using a ring-shaped anisotropic magnetoresistance-based sensor: a model for a magnetoresistance-based biosensor”, Applied Physics Letters, 81(12), 2211-2213, 2002.

[11] R. Hao, R.Xing, Z. Xu, Y.Hou, S. Gao and S. Sun, S., "Synthesis, functionalization, and biomedical applications of multifunctional magnetic nanoparticles", Advanced Materials, 22(25), 2729-2742, 2010.

[12] S.J. Son, J. Reichel, B.He, M.Schuchman and S.B. Lee, "Magnetic nanotubes for magnetic-field-assisted bioseparation, biointeraction, and drug delivery", Journal of the American Chemical Society, 127(20), 7316-7317, 2005.

[13] J. Liu, S.Z. Qiao and Q.H. Hu, "Magnetic nanocomposites with mesoporous structures: synthesis and applications", Small, 7(4), 425443, 2011.

[14] P.K.Jain, X.Huang, I.H.El-Sayed and M.A. El-Sayed, "Noble metals on the nanoscale: optical and photothermal properties and some applications in imaging, sensing, biology, and medicine", Accounts of chemical research, 41(12), 1578-1586, 2008.

[15] L. Wang, J. Luo, M.M. Maye, Q. Fan, Q. Rendeng, M.H. Engelhard and C.J. Zhong, "Iron oxide-gold core-shell nanoparticles and thin film assembly", Journal of Materials Chemistry, 15(18), 1821-1832, 2005.

[16] A. Akbarzadeh, M. Samiei and S. Davaran, S., "Magnetic nanoparticles: preparation, physical properties, and applications in biomedicine", Nanoscale research letters, 7(1), 144, 2012. 
Bhatia et al., Adv. Nan. Res.; Vol. 1 Issue 1, pp: 1-13, 2018

[17] Z. Fan, M. Shelton, A.K.Singh, D.Senapati, S.A. Khan and P.C. Ray, "Multifunctional plasmonic shell-magnetic core nanoparticles for targeted diagnostics, isolation, and photothermal destruction of tumor cells", ACS nano, 6(2), 1065-1073, 2012.

[18] M G.A. Sotiriou, "Biomedical applications of multifunctional plasmonic nanoparticles", Wiley Interdisciplinary Reviews: Nanomedicine and Nanobiotechnology, 5(1), 19-30, 2013.

[19] W.Brullot, V.K. Valev and T. Verbiest, "Magnetic-plasmonic nanoparticles for the life sciences: calculated optical properties of hybrid structures", Nanomedicine: Nanotechnology, Biology and Medicine, 8(5), 559-568, 2012.

[20] K.Simeonidis, C. Martinez-Boubeta, L.Balcells, C. Monty, G. Stavropoulos, M.Mitrakas and M. Angelakeris, "Fe-based nanoparticles as tunable magnetic particle hyperthermia agents", Journal of Applied Physics, 114(10), 103904, 2013.

[21] C.Sun, J.S.Lee and M. Zhang, "Magnetic nanoparticles in MR imaging and drug delivery", Advanced drug delivery reviews, 60(11), 1252-1265, 2008.

[22] D.K.Kim, Y. Zhang, W.Voit, K.V.Rao and M. Muhammed, "Synthesis and characterization of surfactant-coated superparamagnetic monodispersed iron oxide nanoparticles", Journal of Magnetism and Magnetic Materials, 225(1), 30-36, 2001.

[23] Z. Xu, Y.Hou and S. Sun, "Magnetic core/shell $\mathrm{Fe} 3 \mathrm{O} 4 / \mathrm{Au}$ and $\mathrm{Fe} 3 \mathrm{O} 4 / \mathrm{Au} / \mathrm{Ag}$ nanoparticles with tunable plasmonic properties", Journal of the American Chemical Society, 129(28), 8698-8699, 2007.

[24] E.A. Kwizera, E. Chaffin, X. Shen, J. Chen, Q. Zou, Z. Wu, and H. Adhikari, "Size-and Shape-Controlled Synthesis and Properties of Magnetic-Plasmonic Core-Shell Nanoparticles" The Journal of Physical Chemistry C, 120(19), 10530-10546, 2016.

[25] O. Pena-Rodriguez, P.P.Gonzalez Pereza and U.Pal, "MieLab: a software tool to perform calculations on the scattering of electromagnetic waves by multi-layered spheres" International Journal of Spectroscopy, 2011.

[26] P.B. Johnson and R.W. Christy, "Optical constants of the noble metals", Physical Review B, 6(12), 4370, 1972.

[27] P.B.Johnson and R.W. Christy, "Optical constants of transition metals: Ti, v, cr, mn, fe, co, ni, and pd", Physical Review B, 9(12), 5056, 1974. 